\title{
INTERSTELLAR CIRCULAR POLARIZATION AND THE COMPOSITION OF INTERSTELLAR DUST
}

\author{
P. G. MARTIN
}

Institute of Theoretical Astronomy, Madingley Road, Cambridge, England*

\begin{abstract}
This paper shows that optical observations of circular polarization produced by aligned interstellar grains could yield valuable information about the grain material. The interstellar medium is known to be linearly dichroic from observations of interstellar linear polarization; many different grain models using a large variety of compositions can be found to reproduce these observations. Since the same aligned grains make the medium linearly birefringent, a small component of circular polarization can result from incident linearly polarized light if the position angle of the linear polarization does not coincide with either principal axis of the medium. Here calculations are presented to demonstrate that the wavelength of the circular polarization is sensitive to the imaginary part of the complex refractive index of the grain material. This provides an opportunity of investigating whether the grains are characteristically dielectric or metallic. Some possible observations are suggested.
\end{abstract}

\section{Introduction}

It is proposed that observations of circular (elliptical**) polarization from aligned interstellar grains could be useful in placing restrictions on the type of grain material involved. At the present many grain models based on a large variety of dielectric through metallic composition can be found to produce a reasonable fit to the observed wavelength dependence of extinction and linear polarization in the optical region of the spectrum. However over this same range of models widely different circular polarization is predicted; thus circular polarization measurements offer a new method of resolving the longstanding ambiguity in the interpretation of existing observations. This investigation was prompted by a statement near the end of van de Hulst's monograph (1957) forseeing interstellar circular polarization; since then this interesting possibility has not received the attention it probably deserves.

In Section 2 the phenomena of dispersion and extinction in a medium containing interstellar grains and their relation to the Stokes parameters describing circular and linear polarization are reviewed. Next the wavelenght dependence of circular polarization for different materials is investigated using the Mie theory for circular cylinders. Finally several observations are suggested.

\section{Dispersion, Entinction and Polarization}

The action of interstellar grains on radiation passing through interstellar space can be shown to be equivalent to that of a medium with complex refractive index

* Present address: Dept. of Astronomy, University of Toronto, Toronto, Ontaria, Canada.

** The term circular polarization is used to denote the circular component (Stokes parameter $V$ ) of what is generally elliptical polarization. 


$$
\grave{m}=\tilde{n}-i \tilde{k}=1-i(2 \pi)^{-2} \lambda^{3} N S(0),
$$

where $N$ is the number density of grains, $\lambda$ is the wavelength of the light and $S(0)$ is the complex amplitude function of the radiation scattered in the forward direction (van de Hulst, 1957); $\tilde{m}$ is close to once because $N$ is so small. From this formal equivalence the amount of extinction and dispersion can be seen immediately. The linear extinction coefficient $\gamma$ depends on the imaginary part of $\tilde{m}$;

$$
\gamma=4 \pi \lambda^{-1} \tilde{k}=\pi^{-1} \lambda^{2} N \operatorname{Re}[S(0)] \equiv N C_{e},
$$

where $C_{e}$ is the extinction cross-section per grain. The presence of grains also produces a phase lag (or advance if $\varepsilon$ is negative)

$$
\varepsilon=2 \pi \lambda^{-1} s(\tilde{n}-1)=(2 \pi)^{-1} \lambda^{2} N s \operatorname{Im}[S(0)] \equiv \frac{1}{2} N s C_{p},
$$

where $s$ is the pathlength in the medium and $C_{p}$ has been defined by analogy to $C_{e}$.

Both linear and circular polarization depend on interstellar grains of anisotropic shape being aligned so that the averaged profile along the line of sight is non-circular. Observations of linear polarization are usually taken as evidence that such alignment exists, although what alignment mechanism is actually operating is not clearly understood. Except in the direction of the alignment axis of symmetry this medium has different complex refractive indices for orthogonal orientations of the electric vector of the radiation; it is convenient to choose two perpendicular directions, 1 and 2 say, along the long and short principal axes of the grain profile respectively. The symmetry is such that linear dichroism $\left(C_{e 1} \neq C_{e 2}\right)$ and linear birefringence $\left(C_{p 1} \neq C_{p 2}\right)$ can occur, while their circular counterparts cannot. Linear birefringence requires a nonzero imaginary part of the scattering amplitude function (a non-zero phase change). Note that linear birefringence does not necessarily imply linear dichroism (e.g. nonabsorbing particles small compared to the wavelength) or vice-versa (Figure 1). In addition to alignment of the grains and the resulting linear birefringence the incident radiation must be linearly polarized at a position angle other than $0^{\circ}$ or $90^{\circ}$ relative to the principal axes 1 and 2 for the birefringence to produce the desired circular polarization.

Changes in polarization can be described conveniently in terms of the four Stokes parameters $I, Q, U, V$. Here we adopt the convention that for an observer looking towards the source of radiation position angles are measured counterclockwise from axis 2 in the plane perpendicular to the direction of propagation. Thus, for example, $V>0$ corresponds to right-hand circular polarization in which the position angle of the electric vector increases with time (counter-clockwise rotation).

Two useful expressions can be written down from the amplitude-phase definitions of $Q$ and $V$ :

$$
\begin{aligned}
& \mathrm{d} Q=E_{2}^{2}-E_{1}^{2} \simeq \frac{1}{2} I\left[\left(1-\gamma_{2} \mathrm{~d} s\right)-\left(1-\gamma_{1} \mathrm{~d} s\right)\right]=\frac{1}{2} I N \mathrm{~d} s\left(C_{e 1}-C_{e 2}\right) \\
& \mathrm{d} V=2 E_{2} E_{1} \sin \left(\varepsilon_{2}-\varepsilon_{1}\right) \simeq U\left(\varepsilon_{1}-\varepsilon_{2}\right)=\frac{1}{2} N \mathrm{~d} s\left(C_{p 1}-C_{p 2}\right) U .
\end{aligned}
$$

These equations emphasize the following important points: that within the above 


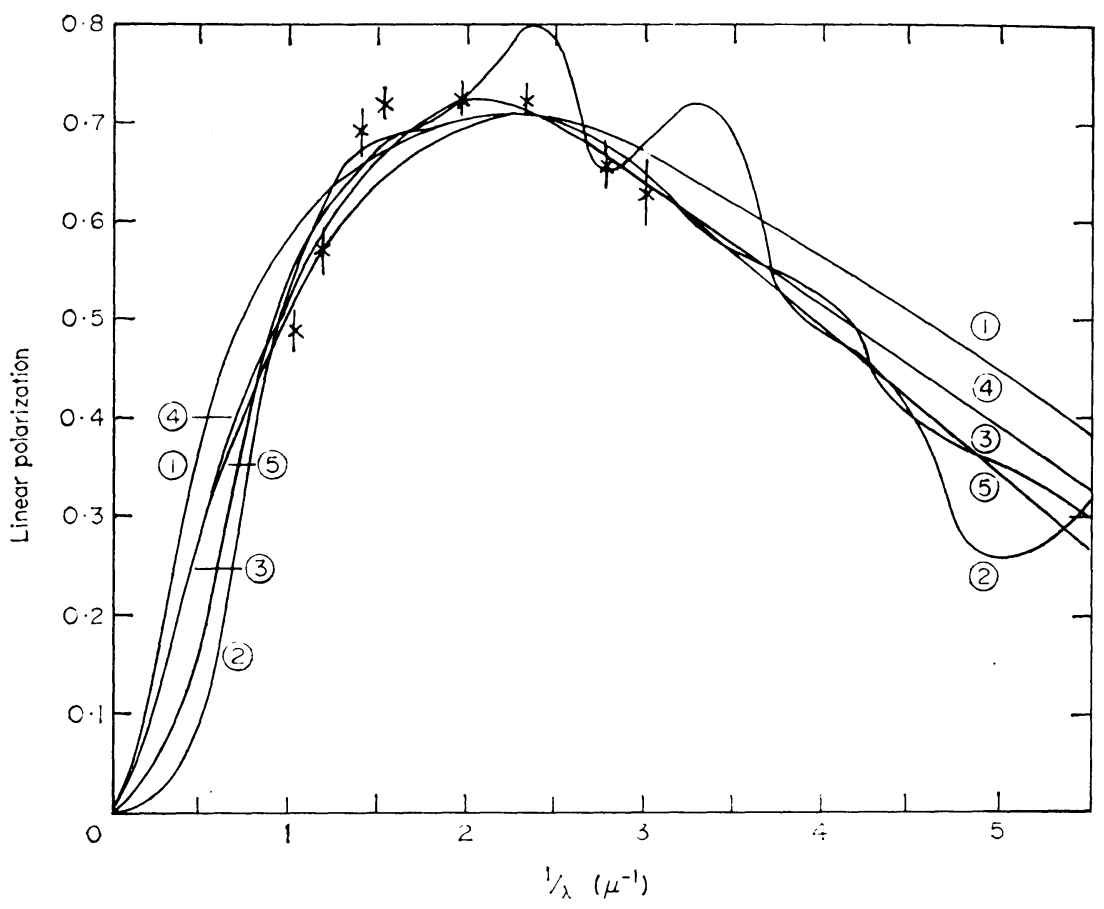

Fig. 1. Wavelength dependence of linear polarization for different complex refractive indices ('picket fence' alignment for long circular cylinders). For each curve the vertical scale and the grain size were chosen to obtain a good approximation to the shape of the mean observed linear polarization (crosses). The vertical scale is arbitrary. $1 m=1.1 ; 2 m=1.5 ; 3 m=1.5-0.1 ; 4 m=$ $1.5-0.5 i ; 5 m=1.5-1.5 i$.

convention interstellar grains produce the $Q$ component of linear polarization, and that a component $V$ results only when the incident radiation has a $U$ component of linear polarization and the medium is linearly birefringent. The latter two requirements must be considered in choosing good observational prospects.

A more general treatment along the lines developed by van de Hulst (1957) and Serkowski (1962) shows that

$$
\begin{aligned}
& I^{-1} \frac{\mathrm{d} I}{\mathrm{~d} s}=-\frac{1}{2} N\left(C_{e 1}-C_{e 2}\right)-\frac{1}{2} N\left(C_{e 1}-C_{e 2}\right)(Q / I) \\
& \frac{\mathrm{d}(Q / I)}{\mathrm{d} s}=\frac{1}{2} N\left(C_{e 1}-C_{e 2}\right)-\frac{1}{2} N\left(C_{e 1}-C_{e 2}\right)(Q / I)^{2} \\
& \frac{\mathrm{d}(U / I)}{\mathrm{d} s}=\frac{1}{2} N\left(C_{p 1}-C_{p 2}\right)(V / I)-\frac{1}{2} N\left(C_{e 1}-C_{e 2}\right)(Q / I)(U / I) \\
& \frac{\mathrm{d}(V / I)}{\mathrm{d} s}=\frac{1}{2} N\left(C_{p 1}-C_{p 2}\right)(U / I)+\frac{1}{2} N\left(C_{e 1}-C_{e 2}\right)(Q / I)(V / I) .
\end{aligned}
$$

Usually only the first term on each right-hand side will be important. 
Since in most observational cases the column density $N \mathrm{~d} s$ is not known (or is model dependent) it is useful to remove this parameter by expressing the predicted components $V$ and $Q$ on the same arbitrary scale and then to find the expected magnitude of $V$ using observations of the linear polarization in that direction. (This latter step would of course give $N \mathrm{~d} s$ for each model if erquired). The ratio of Equations (4) and (5) gives an indication of the size of $V$ to be expected; if $Q$ is $1 \%, C_{p 1}-C_{p 2} \simeq C_{e 1}-C_{e 2}$ and $U / I=0.1$ then $V$ would be $0.1 \%$. The relative phase $\Delta \varepsilon$ would be only 0.01 radians, about two orders of magnitude less than that of a quarter-wave plate.

\section{Wavelength Dependence of Circular Polarization}

To predict the wavelength dependence of $V$, it is necessary to calculate $C_{p}$ on the basis of some model for the interstellar grains. As mentioned above, the size (or size distribution) can be chosen so that a match to the observed wavelength dependence of linear polarization (and extinction) is obtained. Models thus restricted show that $V$ is sensitive to the nature of the grain material, and on this basis it is proposed that observations of $V$ could be a useful discriminant for grain composition.

Many calculations of the linear polarization expected from aligned interstellar grains have used infinite circular cylinders as a model, since for this shape Mie solutions exist, whereas for a general spheroid no such solutions are available. Actually the Mie solutions are valid for finite cylinders of any radius as long as the conditions $l \gg a$ and $l \gg \lambda$ are satisfied (van de Hulst, 1957). Microwave analogue experiments with spheroids and cylinders (Greenberg, 1961; Greenberg et al, 1968) demonstrate qualitative agreement even when $l \simeq 2 a$ (all wavelengths).

The Mie theory for infinite cylinders, including the case of oblique incidence, gives the cross-sections as series expansions (Lind and Greenberg, 1966)

$$
\begin{aligned}
& Q^{E}=\frac{C^{E}}{2 a}=\frac{2}{x}\left(b_{0}^{E}+2 \sum_{n=1}^{\infty} b_{n}^{E}\right) \\
& Q^{H}=\frac{C^{H}}{2 a}=\frac{2}{x}\left(a_{0}^{H}+2 \sum_{n=1}^{\infty} a_{n}^{H}\right),
\end{aligned}
$$

where $x=2 \pi a / \lambda, C=C_{e}+i C_{p}$ and the superscripts $E$ and $H$ identify the two polarizations with electric vectors respectively in and perpendicular to the plane formed by the cylinder axis and the direction of propagation. Both the real and imaginary parts of the complex coefficients have to be calculated when $Q_{s}$, the efficiency factor for scattering, is determined so that usually no new coefficients would have to be calculated for either $Q_{e}$ or $Q_{p}$; only a regrouping of terms is necessary.

A computer program kindly supplied by N. C. Wickramasinghe was appropriately modified to include computations of $Q_{p}$. In Figure 1 some results are shown for models chosen to match the mean observed linear polarization curve tabulated by Coyne and Gehrels (1967). No attempt is made at this point to use a size distribution or oblique incidence since we are interested in isolating the effects of changing the complex re- 
fractive index $m$ of the grain material. Nevertheless even for this simplified calculation for a single grain size and 'picket fence' alignment the agreement with observation is quite reasonable for each complex refractive index. (Averaging over size will smear out the oscillations in the curve(s) for $m=1.5$.)

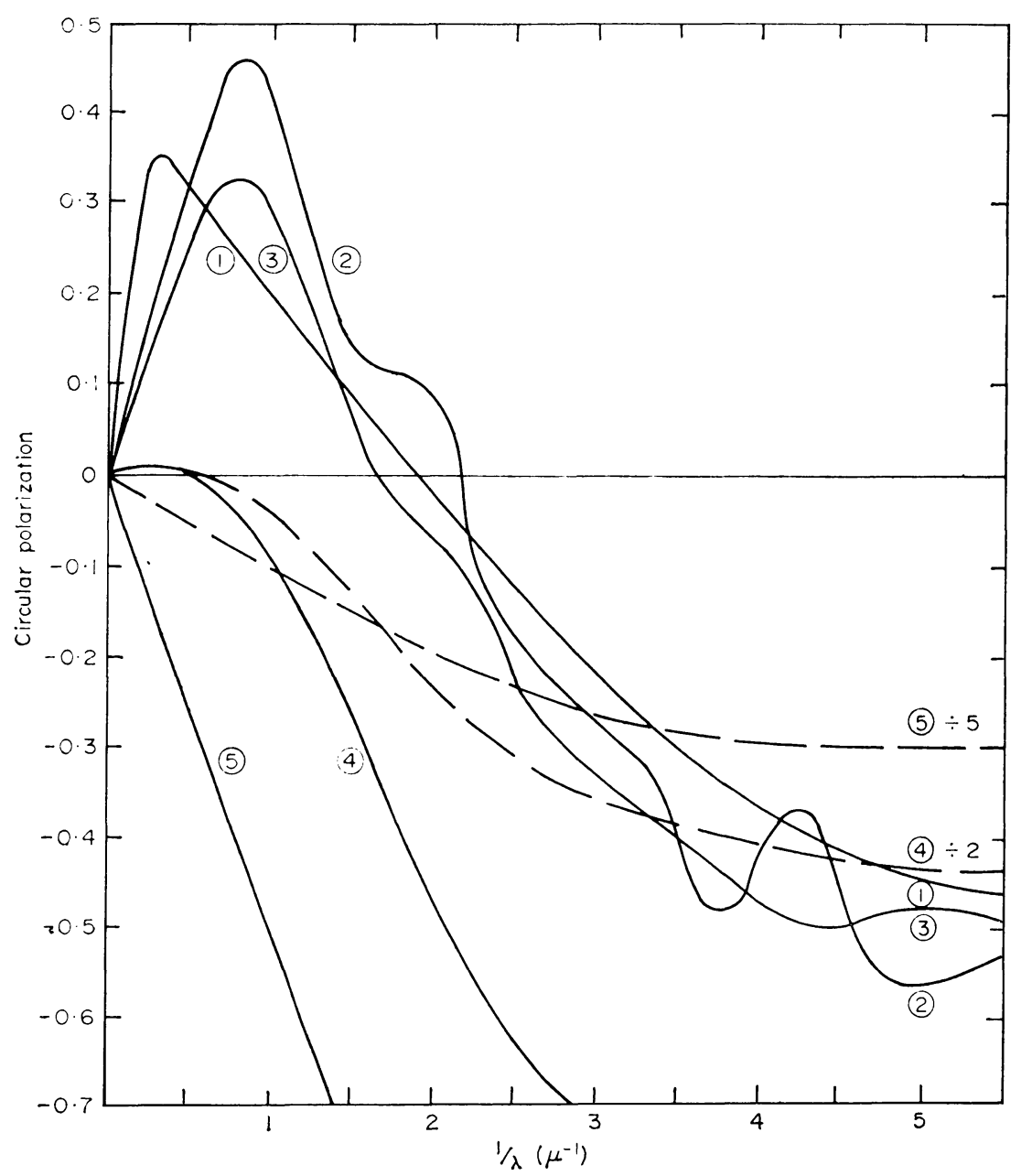

Fig. 2. Wavelength dependence of circular polarization (for constant intrinsic linear polarization) for the grains producing the linear polarization in Figure 1.

What is important is that these different grains which produce similar linear polarization curves in Figure 1 do not have the same circular polarization, as is clear from Figure 2. Evidently changing $n$ has little influence on the wavelength dependence of $V$, but it can be seen that variations in $k$ have a large effect. Curves for the two extremes, $k=0$ and $k \simeq n$, reveal characteristic differences. The former, for dielectric materials, have a relative phase lag a long wavelengths which reverses to an advance in the optical 
region. On the other hand what we shall call 'metallic' materials produce a relative phase advance which increases towards shorter wavelengths. Some insight into these different behaviours can be obtained through use of approximations to the Mie theory (Martin, 1972).

Several different materials have been suggested for the interstellar grains. Of these, ices, dirty ice and silicates are dielectric (not strongly absorbing) whereas graphite and iron are metallic.

\section{Observations}

In conclusion we suggest some observations of $V$ that could be made. As discussed above, observations of interstellar linear polarization can establish that the grains are aligned, the magnitude of the linear polarization $Q$, and the position angle relative to which the incident radiation must have a $U$ component of polarization. It may be necessary to infer this interstellar polarization from observations of stars near the intrinsically polarized source. Sources should be chosen for which both $Q$ (interstellar) and $U$ are reasonably large so that $V$ is within the range of detection. Clearly observations at a number of wavelengths would be desirable in order to make full use of the predictions of the models.

As a specific example we cite the Crab Nebula, which in addition to satisfying the above criteria offers the advantage that changes of the sign of $V$ at different positions in the Nebula can be predicted, due to the variations in position angle that occur. In the past observations have not had sufficient accuracy to detect $V$, but recently some succes in measuring $V$ has been achieved. These observations are discussed in the following paper (Martin, et al., 1972). Other possibilities include stars which show intrinsic linear polarization (latetype stars, Dyck et al., 1971; early-type stars, Serkowski, 1970) and linearly polarized galactic nuclei, if these sources lie behind enough aligned interstellar grains.

Also, as Serkowski (1962) has pointed out, if the direction of grain alignment varies strongly along the line of sight a measurable circular component could result for heavily obscured stars. From the point of view of comparing with theoretical models such observations might be of less value, because the magnitude and sign of the 'incident' polarization $U$ are not known.*

\section{Acknowledgements}

This work was supported through a Commonwealth Scholarship, by the National Science Foundation under grant GP 31356X and by the National Aeronautics and Space Administration under Grant NGR 33-008-102. I thank Prof. R. Novick for his hospitality during my stay at Columbia University. This paper is a brief version of work previously published (Martin, 1972).

* Kemp $(1972,1973)$ has reported detection of this effect. A detailed discussion has been given by Martin (1972). 


\section{References}

Coyne, G. and Gehrels, T.: 1967, Astron. J. 72, 892.

Dyck, H. M., Forrest, W. J., Gillett, F. C., Stein, W. A., Gehrz, R. D., and Woolf, N. J.: 1971, Astrophys. J. 165, 57.

Greenberg, J. M.: 1968, Stars and Stellar Systems 7, 221.

Greenberg, J. M., Pedersen, N. E., and Pedersen, J. C.: 1961, J. Appl. Phys. 32, 233.

Kemp, J. C.: 1972, Astrophys J. Letters 175, L35.

Kemp, J. C.: 1973, this volume, p. 181.

Lind, A. C. and Greenberg, J. M.: 1966, J. Appl. Phys. 37, 3195.

Martin, P. G.: 1972, Monthly Notices Rox Astron. Soc. 159, 179.

Martin, P. G.: 1972, Paper presented at IAU Colloq. 23, Nov. 1972, Tucson, Ariz.

Martin, P. G., Illing, R., and Angel, J. R. P.: 1972, Monthly Notices Roy. Astron. Soc. 159, 191.

Serkowski, K.: 1962, Adv. Astron. Astrophys. 1, 290.

Serkowski, K.: 1970, Astrophys. J. 160, 1083.

Van de Hulst, H. C.: 1957, Light Scattering by Small Particles, Wiley, New York. 